\title{
Search for New Physics through the Reconstruction of Challenging Signatures with the ATLAS detector
}

\author{
Marianna Testa* \\ On behalf of the ATLAS Collaboration \\ LNF-INFN \\ E-mail: marianna.testa@lnf.infn.it
}

Many theories of beyond the Standard Model (BSM) physics predict unique signatures which are difficult to reconstruct and the background rates are also a challenge. Signatures from displaced vertices anywhere from the inner detector to the muon spectrometer as well as those of new particles with fractional or multiple value of the charge of the electron or high mass stable charged particles are experimentally demanding signatures. The results of searches using data collected by the ATLAS detector in $p p$ collisions at $\sqrt{s}=13 \mathrm{TeV}$ are presented

ICHEP 2018, XXXIX Integrantional Confernece on HIgh Energy Physics

4-11 July 2018

COEX, Seoul

${ }^{*}$ Speaker. 


\section{Introduction}

The lack of any deviation from the Standard Model (SM) at the LHC has brought to an increasing interest in the exploration of unusual final state signatures which elude the searches based on prompt signatures and stable particles. Unconventional signature arises from long lived particles (LLP), particles with fractional or multiple value of the electric charge or high mass (meta)stable charged particle. Since the ATLAS detector was not designed for such signatures, their reconstruction and trigger are challenging, and have to face increasing pileup conditions at the LHC in the coming years. In this document recent searches for new phenomena, mostly related to LLPs, leading to unconventional signatures in the ATLAS detector [1] are presented.

\section{Search for long lived particles}

A search of short tracks (tracklets), which leave hits only in the innermost Inner Detector (ID) layers, have been performed in [2]. The benchmark model is the decay of a chargino $\widetilde{\chi_{ \pm}}$ into a low momentum pion, which is not detectable, and a neutralino $\tilde{\chi}_{0}$ (see Fig.1, left). The charginos are prodcuted either via a direct electroweak $(\mathrm{EW})$ gaugino production or via gluino pair (GP) production, with $m \widetilde{\chi_{ \pm}}-m \widetilde{\chi_{0}} \sim \mathrm{O}(100 \mathrm{MeV})$. The events are required to have high missing transverse energy (MET), a high quality tracklet, no leptons and at least one high $p_{\mathrm{T}}$ jet from intial state radiation. Tracklets used in this analysis are reconstructed using hits not associated with
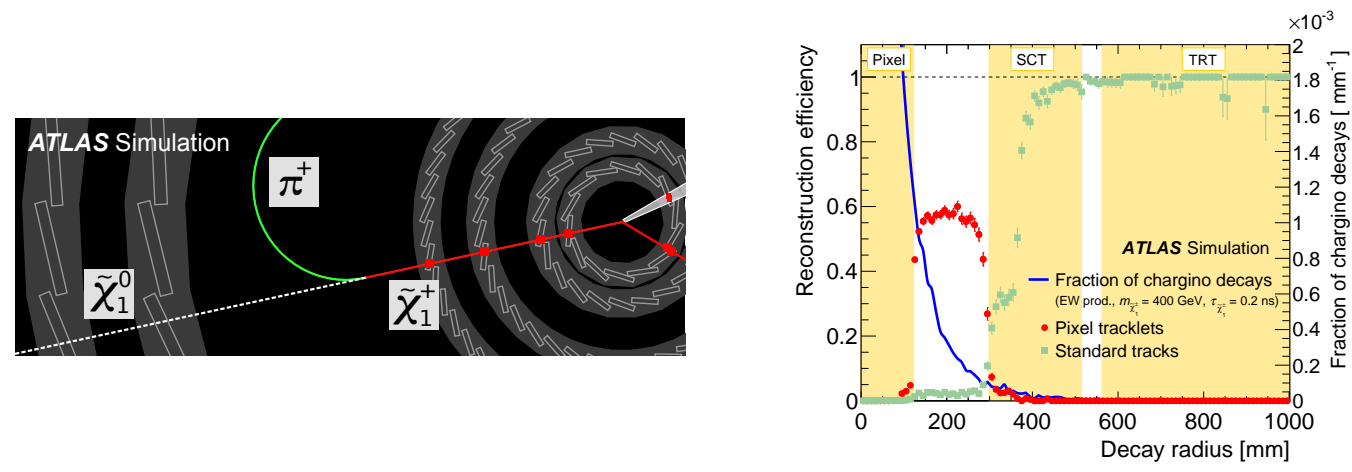

Figure 1: Left: $p p \rightarrow \widetilde{\chi}_{1}^{+} \widetilde{\chi}_{1}^{-}+$jet event, with long-lived charginos. The $\widetilde{\chi}_{1}^{+}$decays into a low-momentum pion and a $\widetilde{\chi}_{1}^{0}$ after leaving hits in the four pixel layers (indicated by red makers). Right: Chargino reconstruction efficiency as a function of decay radius. The reconstruction efficiency of pixel tracklets before applying the fake-rejection criteria is shown in red, while that obtained with the standard tracking algorithm is shown in green. Plots are taken from [2].

standard tracks. More than 3 hits in the pixel layers and no hits in the semiconductor tracker layers are required. The efficiency of tracklets reconstruction is shown in Fig.1, right. This analysis is sensitive to lifetime of LLPs from 10ps to $10 \mathrm{~ns}$. The background contribution from $t \bar{t}$ and $W+$ jets events has been evaluated from data. No excess of candidate events in the $p_{\mathrm{T}}$ distribution of pixel tracklets has been found. Improved limits with respect to Run1 analysis on the lifetime and mass of the chargino have been set. More details can be found in [1]. Challenging tracks reconstruction has been used also in the search of LLPs decaying in the ID away from the primay vertex, giving raise to displaced vertices (DV). Such a signature accompanied by high MET from $\widetilde{\chi}^{0}$ has been searched 
for in [3]. The DV signature characterizes the decay of LLPs with lifetimes of $10^{-12}-10^{-9} \mathrm{~s}$. The benchmark model is a "R-hadron", arising when a gluino has a lifetime larger than $10 \mathrm{ps}$ and form a bound state, decaying into jets and neutralinos. Candidate events are selected requiring a trigger on MET, with a high-mass and a multi-track DV. A DV is reconstructed using tracks reconstructed from the standard and the large-radius tracking (LRT) algorithm. This latter uses relaxed requirements in number of silicon hits and impact parameter of the candidate tracks. Using the LRT a significant increase of the vertex reconstruction efficiency on the signal is obtained, as shown in Fig.2, left. A few background events come from hadronic interactions, random crossing
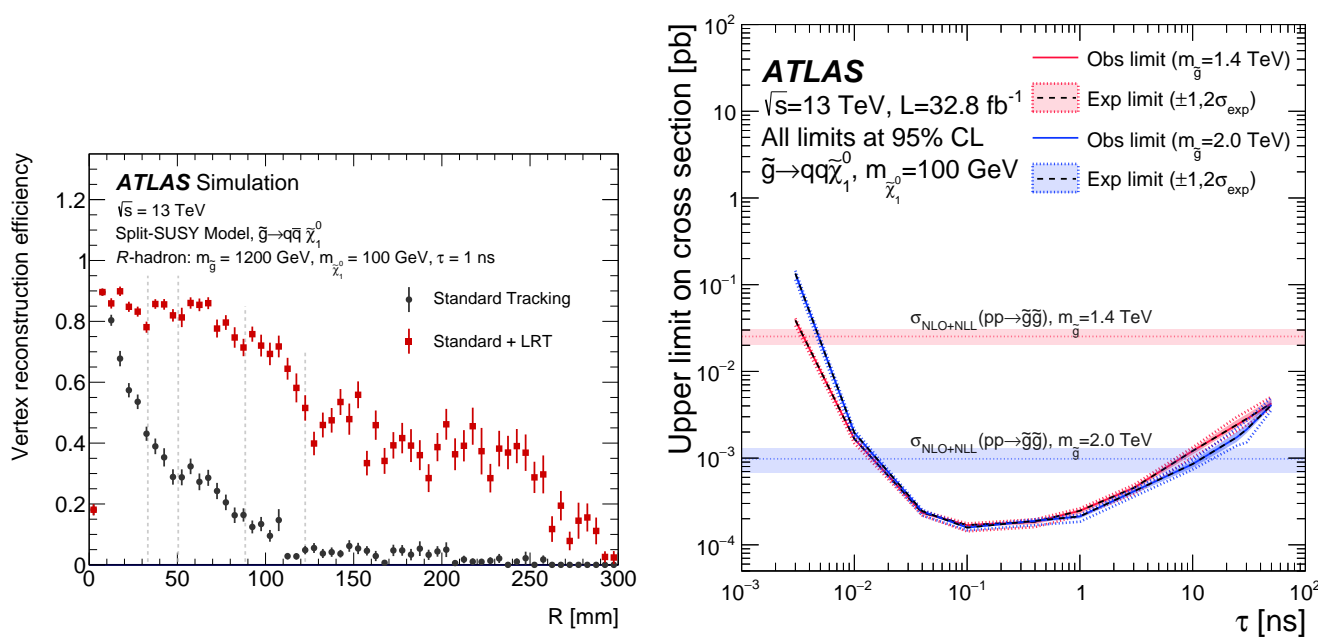

Figure 2: Left: vertex reconstruction efficiency as a function of radial position $\mathrm{R}$ with and without the special Large Radius Tracking processing [3]. Right:Upper 95\% CL limits on the signal cross section are shown in (a) for $m \widetilde{g}=1400 \mathrm{GeV}$ and $m \widetilde{g}=2000 \mathrm{GeV}$ as a function of lifetime $\tau$, for fixed $m \chi_{1}^{0}=100 \mathrm{GeV}$ [3].

tracks and merged vertices and are estimated using data-driven methods. No excess has been observed and limits on the cross section have been set as a function of the R-hadron lifetime, as shown in Fig. 2 right.

A search of massive charged R-Hadrons LLP with low $\beta$, predicted in many BMS modes, has been perfomed in [4]. The signature is characterized by a high specific ionization loss in the pixel system. The benchmark model is the decay of a gluino R-hadron, stable or meta-stable with lifetime of $\mathrm{O}(1-10) n s$, to $q \bar{q} \widetilde{\chi}_{0}$. A significant improvement of the sensitivity of this analysis on Run 2 data has been obtained thanks to the insertable B-Layer [5] installed for Run2, has been obtained. No excess has been found in the distribution of the mass of the selected candidate particles.

A search for neutral LLPs has been also performed. Many BSM models predict a dark sector weakly coupled with the SM photon, where the dark photons are mixed with SM photons. If the the dark photons are long lived and have low mass, they decay in collimated pairs of leptons and/or light hadrons (displaced lepton jets). In Ref. [6] at least two displaced jets are searched either only in the Muons Spectrometer (MS) or both in the MS and in the hadronic calorimeter. Dedicated high level trigger have been used to select two close-by muons. No excess has been found: limits on dark photon life time for the used benchmark mode (FRVZ model [7]) have been set. Neutral LLPs decays in the hadronic calorimeter have been searched in [8]. These LLPs arise in BSM models, where the dark sector is weakly coupled with the SM through a heavy neutral 

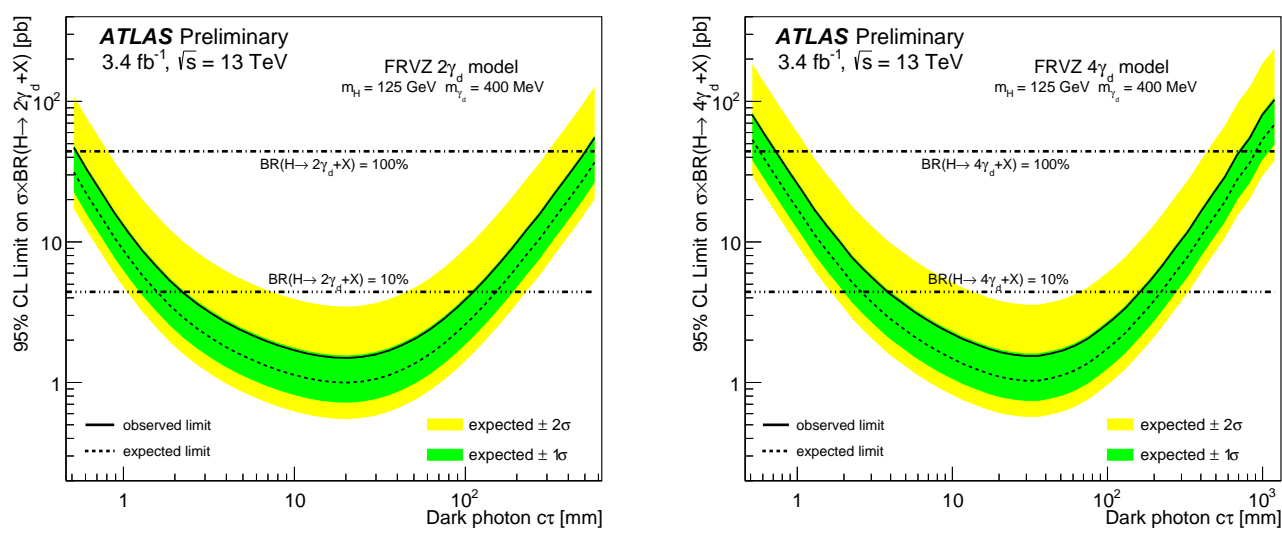

Figure 3: The $95 \%$ upper limits on the $\sigma \times \mathrm{BR}$ for the FRVZ $125 \mathrm{GeV}$ Higgs decaying to two (four) dark photons $+\mathrm{X}$ benchmark models as a function of the dark photon lifetime (c $\tau$ ), in the left (right) figure. The horizontal lines correspond to $\sigma \times \mathrm{BR}$ for two values of the BR of the Higgs boson decay to dark photons. Plots are taken from [6].

boson. This latter decays into long lived scalar particles which decay into SM fermions hadronising in jets. The two jets are required to have high ratio of the hadronic over electromagnetic released energy, no tracks associated and a small width inside the hadronic calo. No excess of candidate events has been found and limits on the LL scalar particle have been set.

\section{References}

[1] ATLAS Collaboration, The ATLAS Experiment at the CERN Large Hadron Collider, JINST 3 (2008) S08003

[2] ATLAS Collaboration, Search for long-lived charginos based on a disappearing-track signature in pp collisions at $\sqrt{s}=13 \mathrm{TeV}$ with the ATLAS detector, JHEP 06 (2018) 022

[3] ATLAS Collaboration, Search for long-lived, massive particles in events with displaced vertices and missing transverse momentum in $\sqrt{s}=13 \mathrm{TeV}$ pp collisions with the ATLAS detector, Phys. Rev.D 97 (2018) 052012

[4] ATLAS Collaboration, Search for metastable heavy charged particles with large ionization energy loss in pp collisions at $\sqrt{s}=13 \mathrm{TeV}$ using the ATLAS experiment, Phys. Rev. D 93, 112015 (2016)

[5] ATLAS Collaboration, ATLAS Insertable B-Layer Technical Design Report, Tech. Rep. CERN-LHCC-2010-013, ATLAS-TDR-19

[6] ATLAS Collaboration, Search for long-lived neutral particles decaying into displaced lepton-jets in proton-proton collisions at $\sqrt{s}=13 \mathrm{TeV}$ with the ATLAS detector, ATLAS-CONF-2016-042, https://atlas.web.cern.ch/Atlas/GROUPS/PHYSICS/CONFNOTES/ATLAS-CONF-2016-042

[7] A. Falkowski et al., Discovering Higgs Decays to Lepton Jets at Hadron Colliders, Phys. Rev. Lett. 105 (2010) 241801

[8] ATLAS Collaboration, Search for long-lived neutral particles decaying in the hadronic calorimeter of ATLAS at $\sqrt{s}=13 \mathrm{TeV}$ in $3.2 \mathrm{fb}^{-1}$ of data, ATLAS-CONF-2016-103, https://atlas.web.cern.ch/Atlas/GROUPS/PHYSICS/CONFNOTES/ATLAS-CONF-2016-103 